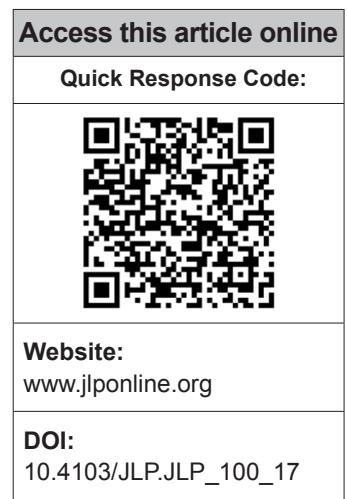

Department of Medicine, Jawaharlal Nehru Medical College, DMIMSU, Wardha, Maharashtra, India

Address for correspondence:

Dr. Sachin Agrawal, Department of Medicine,

Jawaharlal Nehru Medical College, DMIMSU, Wardha, Maharashtra, India. E-mail: dragrawal82@ gmail.com

Submission: 23-07-2017 Accepted: 16-10-2017

\title{
Leukocyte count: A reliable marker for the severity of organophosphate intoxication?
}

\author{
Sunil Kumar, Sachin Agrawal, Nitin Raisinghani, Shameem Khan
}

\section{Abstract:}

INTRODUCTION: Organophosphorus poisoning (OPP) is a major public health problem in developing countries like India. Leukocyte count is a simple and inexpensive test, and elevated count is associated with acute inflammation and increased oxidative stress-like OPP. This study was done to correlate the severity of acute OPP with leukocyte count and also to assess the prognosis.

MATERIALS AND METHODS: A prospective, observational clinical study was done on 80 patients suspected of OPP of age $>15$ years admitted to emergency unit at a tertiary rural teaching health-care center of Central India. Serum cholinesterase level and leukocyte count were estimated at the time of admission in all patients and severity of OPP was assessed according to Peradeniya organophosphorus poisoning (POP) scale.

RESULTS: The mean age of the patients was 33.52 years (standard deviation [SD] 11.62) in males and 27.30 years (SD 7.33) in females. Among them, $57(71.25 \%)$ were males and $23(28.75 \%)$ were females. The severity of poisoning was directly correlated with serum cholinesterase level $(P=0.0001)$. Leukocyte count had a sensitivity of $60 \%$, specificity of $76 \%$, and negative predictive value of $85 \%$ if counts were more than 12,000 and $30 \%$ sensitivity, $95 \%$ specificity, and $80 \%$ negative predictive value if counts were more than 15,000 in predicting mortality in patients with OPP.

CONCLUSION: Leukocyte count levels on admission can be used a prognostic marker in patients with OP poisoning.

Key words:

Correlation, leukocyte count, organophosphorus, plasma cholinesterase, poisoning, severity

\section{Introduction}

P oisoning with organophosphorus (OP) compounds is an important global health problem having more concern in Vidarbha region of Maharashtra in India. This region is home to approximately 3.4 million cotton farmers, and $95 \%$ of these are struggling with the massive debt. Majority of poisoning cases are from agriculture areas due to easy availability of OP compounds combined with their sale over the counter. ${ }^{[1]}$ Laboratory evidence of OPP is usually confirmed by measuring the decreases in the acetylcholine esterase activity.

This is an open access article distributed under the terms of the Creative Commons Attribution-NonCommercial-ShareAlike 3.0 License, which allows others to remix, tweak, and build upon the work non-commercially, as long as the author is credited and the new creations are licensed under the identical terms.

For reprints contact: reprints@medknow.com
Leukocytosis in stress like injury, poisoning is due to neutrophilia caused by neutrophil margination, and not due to increased marrow production. Normally, they are produced in the bone marrow and comprise approximately $60 \%$ of the blood. These cells are critically important to an immune response and migrate from the blood to tissues during an infection and stress-like poisoning. Theoretically, patients with significant stress should have a higher degree of leukocytosis compared to patients with minor stress. ${ }^{[2]}$ Since the complete blood count is one of the most common tests obtained in Intensive Care Unit (ICU) patients, leukocytes level could serve as an easy-to-obtain marker for severity of

How to cite this article: Kumar S, Agrawal S, Raisinghani N, Khan S. Leukocyte count: A reliable marker for the severity of organophosphate intoxication?. J Lab Physicians 2018;10:185-8. 
organophosphorus poisoning (OPP). In centers where the measurement of cholinesterase activity cannot be done, management is based on the assessment of severity of intoxication, which depends largely on clinical findings and basic blood parameters such as leukocyte count. The aim of this study was to determine whether the monitoring of number of leukocytes may be may be taken as a marker for the severity of OPP.

\section{Materials and Methods}

A prospective observational study was done on 80 patients, in ICU of the Medicine Department of Acharya Vinoba Bhave rural hospital, attached with Jawaharlal Nehru Medical College in Wardha district of Vidarbha region of Central India for 1 year from October 2014 to September 2015, after clearance from institutional ethical committee. All consecutive patients were enrolled in study after taking consent from each patient/relative for obtaining medical history and clinical examination. Blood samples were collected at the time of admission for laboratory investigations such as complete blood count as well as plasma ChE. Diagnoses of acute OP poisoning were done on the basis of the history of intake or exposure to OP compound and seeing the packet/container consumed by the patients, combined with the characteristic symptoms of cholinergic and muscarinic toxidrome.

\section{Data collection}

Patients with double insecticide/multiple poisoning with other drugs such as opioids, diazepam, and alcohol were excluded. Apart from the routine and detailed clinical examination, assessment was also done based on the Peradeniya organophosphorus (POP) scaling system, ${ }^{[3]}$ which included pupil size, respiratory rate, pulse rate, level of consciousness of the patient, and the presence or absence of convulsion and fasciculation. A score of $0-3$ was considered as mild poisoning, 4-7 as moderate poisoning, and 8-11 as severe poisoning. Severity of poisoning based on clinical symptoms was classified into mild, moderate, and severe. ${ }^{[4]}$ Patient's venous blood samples were taken for plasma ChE level and Christmas Bird Count.

Plasma ChE was measured by cholinesterase butyrylthiocholine, kinetic method (Biological ref. range $5000-12,000 \mathrm{U} / \mathrm{L}$ at $\left.37^{\circ} \mathrm{C}\right)$. Instrument used was Beckman AU 480 analyzer.

Cholinesterase catalyzes the hydrolysis of S-butyrylthiocholine iodide to thiocholine iodide and butyrate. Thiocholine iodide reacts with 5,5'-dithiobis2-nitrobenzoate and forms the yellow-colored product 5-mercapto-2-nitrobenzoate. The rate of formation of this product is directly proportional to the catalytic cholinesterase activity. Complete blood cell count was done by hematology analyzer counter report machine (HORIBA ABX, Horiba/Pentra XL80, HORIBA Medical USA). Parameters used in this machine are complete blood pictures such as white blood cell count, red blood cell count, hemoglobin, hematocrit, mean corpuscular volume, mean cell hemoglobin, mean corpuscular hemoglobin concentration, red cell distribution width, platelet count, mean platelet volume, platelet crit, and platelet distribution width.

\section{Statistical analysis}

Statistical analysis was done using descriptive and inferential, using one-way ANOVA, Student's unpaired $t$-test, and Pearson's correlation coefficient. Continuous data were summarized as mean \pm one standard deviation (SD) and were compared using the Student's $t$-test. Categorical data were compared using the Chi-square test. Sensitivity, specificity, and negative predictive values were calculated by software SPSS 22.0 version (Armonk, New York, USA: IBM Corp). The value of $P<0.05$ was considered as level of significance.

\section{Observation and results}

A total of 80 patients were taken for the study. Among them, $57(71.25 \%)$ were male and $23(28.75 \%)$ were female. The mean age of the patients was 31.73 years (SD 10.90). Occupation wise, farmers were on top of the list $37(46.25 \%)$. The most common motive of poisoning was suicidal $60(75 \%)$, followed by accidental $20(25 \%)$. Twenty patients were in severe poisoning with POP scale of 8-11. Baseline characteristics of the patients are shown in Table 1. Total number of patients who survived was 60 whose plasma ChE level was $3287.16 \pm 2719.30$ and the number of nonsurvivors was 20 with plasma $\mathrm{ChE}$ value of $1456.05 \pm 1159.42(P<0.005)$. The mean Acute Physiology and Chronic Health Evaluation (APACHE) score in survivor patients was $10.70 \pm 3.22$ and $21.40 \pm 2.96$ in nonsurvivors $(P<0.0001)$ [Table 2]. Pearson's correlation coefficients showing correlation between plasma $\mathrm{ChE}$ and leukocyte counts with the severity of OPP are shown in Tables 3 and 4 and Figure 1. Of the 80 patients, $26(32.5 \%)$ had leukocytosis on admission. We divided patients of leukocytosis in three groups more than 12,000 , more than 15,000, and more than 20000 for sensitivity and specificity. This showed a sensitivity of $60 \%$, specificity of $76 \%$, and a negative predictive value of $85 \%$ if counts were more than 12,000 ; a 30\% sensitivity, $95 \%$ specificity, and $80 \%$ negative predictive value if counts were more than 15,000; and 10\% sensitivity, $98 \%$ specificity, and $76 \%$ negative predictive value if counts were more than 20,000 in predicting mortality in patients with organophosphate insecticide (OPI) poisoning [Table 5].

\section{Discussion}

Among patients having even mild OPP, presenting to 
the ICU tended to have elevated or at least high normal leukocyte values, suggesting that the stress of the poisoning itself can result in marked demargination. It is clear from this study that leukocyte levels falling within the three ranges can be used to rule out severe poisoning.

In this study, among 80 patients of acute OPP, 57 (71.25\%) were males and $23(28.75 \%)$ were female. People from the young age group between 15 and 29 years (53.75\%) and between 30 and 44 years (30\%) were the most sufferers. These age groups are the most active ones, physically, mentally, and socially, and so, it was more prone to stress during life. Farmers (46.25\%) were the most sufferers in this study. Similar observations were also made by Joshi et al. and Shakuntala and Yogesh, who showed that $51.07 \%$ and $70.07 \%$ were farmers, respectively. ${ }^{[5,6]}$ Acute pesticide exposure can be accidental or suicidal, occupational, bystander exposure, or exposure because of consumption of food items containing pesticide residues. The most common incidence of OPP was suicidal attempt.

In this study, out of 80 patients, 29 were classified as mild poisoning, 31 as moderate poisoning, and 20 as severe as per POP scale. In mild OP poisoning, the mean plasma ChE level was $4930.06 \pm 2989.64$, in moderate poisoning level, it was $2172.61 \pm 972.14$, and in severe poisoning, it was $801.40 \pm 721.93$. Our study observed a significant

Table 1: Baseline characteristics of the patients $(n=80)$

\begin{tabular}{lc}
\hline Variables & Total \\
\hline Age (years) & $31.73 \pm 10.90$ \\
Sex (\%) & $57(71.25)$ \\
Male & $23(28.75)$ \\
Female & \\
Literacy status (\%) & $38(47.5)$ \\
$\quad$ Educated & $42(52.5)$ \\
Illiterate & \\
Occupation (\%) & $37(46.25)$ \\
Farmer & $12(15)$ \\
Labor & $31(38.75)$ \\
Others & \\
Intention (\%) & $60(75)$ \\
Suicidal & $20(25)$ \\
Homicidal & \\
POP scale & 29 \\
0-3 (mild) & 31 \\
4-7 (moderate) & 20 \\
8-11 (severe) & $9882.62 \pm 4682.82$ \\
Leukocyte count &
\end{tabular}

correlation between the degree of derangement in plasma ChE level and severity of OPP ( $P$ value was 0.0001 ). The higher the score on the POP scale, the higher was the degree of derangement in the plasma ChE level. Similar observation was made by Rehiman et al. and Prakash et al. ${ }^{[7,8]}$ In our study, the APACHE II score was also correlated with outcome and it was statistically significant, $P<0.005$, an APACHE II score $>10$ predicted a poor outcome.

Of the 80 patients in this study, 26 (32.5\%) had leukocytosis on admission which showed a sensitivity of $60 \%$, specificity of $76 \%$, and a negative predictive value of $85 \%$ if counts were more than 12,$000 ; 30 \%$ sensitivity, $95 \%$ specificity, and a $80 \%$ negative predictive value if counts were more than 15,000; and 10\% sensitivity, $98 \%$ specificity, and $76 \%$ negative predictive value if counts were more than 20,000 in predicting mortality in patients with OPI poisoning. Leukocytosis and its importance in OPP had been noted in a single study and incidence was found up to $43 \%$. ${ }^{[9]}$

The leukocytosis rate was found to be $76 \%$ in a study by Cander et al. ${ }^{[10]}$ However, a statistically significant correlation was not found between leukocyte levels and length of stay or mortality, although there were higher leukocyte levels in patients who died.

According to our findings, the monitoring of the leukocyte number in conjunction with clinical signs may be helpful in the assessment of the prognosis in centers where the measurement of cholinesterase activity is limited due to financial constraint.

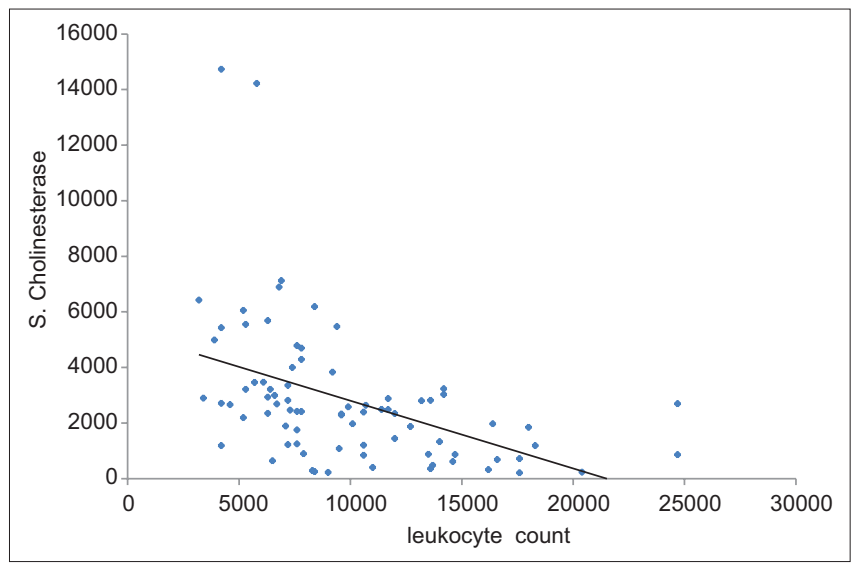

Figure 1: Scatter diagram showing linear correlation between leukocyte count and serum cholinesterase

Table 2: Level of enzymes and its outcome

\begin{tabular}{|c|c|c|c|c|}
\hline Enzymes & Survivors (60) & Nonsurvivors (20) & $t$ & $\boldsymbol{P}$ \\
\hline Serum cholinesterase (mean \pm SD) & $3287.16 \pm 2719.30$ & $1456.05 \pm 1159.42$ & 2.91 & 0.005 \\
\hline APACHE II (mean $\pm S D)$ & $10.70 \pm 3.22$ & $21.40 \pm 2.96$ & 13.11 & 0.0001 \\
\hline
\end{tabular}

$\mathrm{SD}=$ Standard deviation, APACHE II = Acute Physiology and Chronic Health Evaluation II 
Table 3: Pearson's correlation coefficient between serum cholinesterase and leukocyte count

\begin{tabular}{lrccc}
\hline & Mean \pm SD & $n$ Correlation $r$ & $\boldsymbol{P}$ \\
\hline Leukocyte count & $9882.62 \pm 4682.82$ & 80 & -0.448 & $0.0001, \mathrm{~S}$ \\
Serum cholinesterase & $2829.38 \pm 2546.07$ & 80 & & \\
\hline
\end{tabular}

$\mathrm{SD}=$ Standard deviation, $\mathrm{S}=$ Significant

Table 4: Correlation between leukocyte count with severity of organophosphorus poisoning

\begin{tabular}{lccccc}
\hline Severity of OPP & $\boldsymbol{n}$ & Mean \pm SD & SE & $\boldsymbol{F}$ & $\boldsymbol{P}$ \\
\hline Mild & 29 & $7041.37 \pm 2405.42$ & 446.67 & 15.24 & $0.0001, \mathrm{~S}$ \\
Moderate & 31 & $10245.48 \pm 4392.69$ & 788.95 & & \\
Severe & 20 & $13440.00 \pm 5130.95$ & 1147.31 & & \\
Total & 80 & $9882.62 \pm 4682.82$ & 523.55 & & \\
\hline
\end{tabular}

OPP = Organophosphorus poisoning, SD = Standard deviation,

$\mathrm{SE}=$ Standard error, $\mathrm{S}=$ Significant

Table 5: Sensitivity and specificity of mortality in organophosphorus poisoning patients in relation to level of white blood cell count

\begin{tabular}{lccc}
\hline & \multicolumn{3}{c}{ WBC count (\%) } \\
\cline { 2 - 4 } & $>12,000$ & $>15,000$ & $>20,000$ \\
\hline Sensitivity & 60 & 30 & 10 \\
Specificity & 76 & 95 & 98 \\
Positive predictive value & 46 & 66 & 66 \\
Negative predictive value & 85 & 80 & 76 \\
\hline
\end{tabular}

$\mathrm{WBC}=$ White blood cell

One of the major limitations of this study is small sample size and hospital study, so this cannot be generalized to all the population and society. More research is required in this direction.

\section{Conclusion}

While leukocyte counts alone cannot be used to effectively rule in or rule out severe poisoning, its moderate ability to discriminate between patients with and without serious poisoning suggests that it may contribute meaningfully to the disposition decisions. Further study is required to clarify its value in this regard.

\section{Financial support and sponsorship}

Nil.

\section{Conflicts of interest}

There are no conflicts of interest.

\section{References}

1. Behere PB, Behere AP. Farmers' suicide in Vidarbha region of Maharashtra state: A myth or reality? Indian J Psychiatry 2008;50:124-7.

2. Abramson N, Melton B. Leukocytosis: Basics of clinical assessment. Am Fam Physician 2000;62:2053-60.

3. Senanayake N, de Silva HJ, Karalliedde L. A scale to assess severity in organophosphorus intoxication: POP scale. Hum Exp Toxicol 1993;12:297-9.

4. Namba T, Nolte CT, Jackrel J, Grob D. Poisoning due to organophosphate insecticides. Acute and chronic manifestations. Am J Med 1971;50:475-92.

5. Joshi SC, Prakash C, Joshi A, Joshi G. Profile of organophosphorus poisoning at tertiary care hospital in Uttarakhand. J Indian Acad Forensic Med 2013;35:346-8.

6. Shakuntala S, Yogesh G. Analysis of organophosphorus poisoning, at tertiary care hospital: A report. J Evid Based Med Healthc 2015;2:421-30.

7. Rehiman S, Lohani SP, Bhattarai MC. Correlation of serum cholinesterase level, clinical score at presentation and severity of organophosphorous poisoning. JNMA J Nepal Med Assoc 2008;47:47-52.

8. Prakash M, Ram O, Shah Harsh D. Acute organophosphorus poisoning and clinical admission score association among patients admitted in emergency ward of a tertiary teaching hospital of medical college. J Pharm Biomed Sci 2012;17:1-5.

9. Guven M, Dogukan A, Taskapan H, Cetin M. Leukocytosis as a parameter in management of organophosphate intoxication. Turk J Med Sci 2000;30:499-500.

10. Cander B, Dur A, Yildiz M, Koyuncu F, Girisgin AS, Gul M, et al. The prognostic value of the Glasgow coma scale, serum acetylcholinesterase and leukocyte levels in acute organophosphorus poisoning. Ann Saudi Med 2011;31:163-6. 